\title{
Emerging multifaceted roles of BAP1 complexes in biological processes
}

\author{
Aileen Patricia Szczepanski ${ }^{1}$ and Lu Wang ${ }^{1}$
}

\begin{abstract}
Histone H2AK119 mono-ubiquitination (H2AK119Ub) is a relatively abundant histone modification, mainly catalyzed by the Polycomb Repressive Complex 1 (PRC1) to regulate Polycomb-mediated transcriptional repression of downstream target genes. Consequently, H2AK119Ub can also be dynamically reversed by the BAP1 complex, an evolutionarily conserved multiprotein complex that functions as a general transcriptional activator. In previous studies, it has been reported that the BAP1 complex consists of important biological roles in development, metabolism, and cancer. However, identifying the BAP1 complex's regulatory mechanisms remains to be elucidated due to its various complex forms and its ability to target non-histone substrates. In this review, we will summarize recent findings that have contributed to the diverse functional role of the BAP1 complex and further discuss the potential in targeting BAP1 for therapeutic use.
\end{abstract}

\section{Facts}

- The BAP1 complex is an evolutionary conserved, multiprotein complex that functions as a general transcriptional activator via deubiquitination of H2AK119Ub and other epigenetic/transcription factors.

- Variations within the composition of subunits encompassing these BAP1 complexes can determine context-specific or tissue-specific functions. Being universally expressed, the BAP1 complex and its associated subunits play a critical and fundamental role in maintaining development, metabolic processes, and tumorigenesis.

- Both loss-of-function and gain-of-function mutations within these BAP1 complexes have been identified in both developmental diseases and cancers, indicating the need for fine-tune adjustments in the BAP1 complex machinery in order to sustain appropriate

\footnotetext{
Correspondence: Lu Wang (lu.wang1@northwestern.edu)

${ }^{1}$ Simpson Querrey Center for Epigenetics and the Department of Biochemistry and Molecular Genetics, Northwestern University Feinberg School of Medicine, 303 East Superior Street, Chicago, IL 60611, USA

Edited by Ivano Amelio
}

levels for catalytic activity and accurate cellular localization, which are critical for the determinant of cell fate and transformation. The BAP1 complex has emerged as an ideal therapeutic target for treatments involving developmental/metabolic diseases and human cancers.

\section{Open questions}

- What is the mechanism behind the recruitment of the BAP1 complex to chromatin, and which factors determine its chromatin binding specificity?

- What is the crystal structure of the human BAP1 complex?

- What are additional factors associated with functional compositions of these BAP1 complexes in both nuclei and cytosol? Does BAP1 require other subunits to be functional in the cytosol vs. nuclei?

- What are the function and tissue specificity between different compositions of these BAP1 complexes?

- Do all the BAP1 substrates contain similar or identical BAP1-binding motifs, and/or conserved lysine residues? 


\section{Compositions of the BAP1 complex}

Ubiquitin (Ub) is a highly conserved, stable, and ubiquitously expressed protein found in all tissues and eukaryotic organisms ${ }^{1,2}$. The Ub system is significant due to its central role in the maintenance of nearly all cellular processes. However, one way to regulate $\mathrm{Ub}$ activity is within the Ub-dependent proteasome pathway, which includes enzymes such as deubiquitinases (DUBs) that function to catalyze the irreversible conjugation process of $\mathrm{Ub}$ or Ub-like proteins from substrates ${ }^{3}$. The human genome encodes approximately 100 DUB enzymes belonging to seven different families, which exhibit distinct but overlapping cleavage preferences ${ }^{4}$ - such as the ubiquitin C-terminal hydrolase (UCH) subclass, which consists of four members (UCHL1, UCHL3, UCHL5, and BAP1) that share close homology in their catalytic domain ${ }^{5}$. Here on, we will focus on BAP1's functional role in its complex form.

Initially, BAP1 was identified as a BRCA1-associated protein that interacts with the BRCA1-RING finger domain ${ }^{6}$. Further analysis indicated that BAP1 is a novel 729 amino acid-length protein that consisted of a functional domain within 240 amino acids of the aminoterminal, which showed significant homology to other known thiol proteases of the UCH family (Fig. 1A) ${ }^{7}$. Evolutionarily speaking, BAP1 has conserved functional origins going as far back into invertebrate models, such as
Drosophila ${ }^{8}$. In Drosophila, a previously uncharacterized gene, known as Calypso, encoded for a 471 amino acids long, polypeptide chain-which was found to be closely related to the BAP1 human homolog. Through affinity purification methods, Calypso was then found to be copurified with a PcG protein, known as Additional sex combs (ASX) by Müller's lab ${ }^{8}$ (Fig. 1B). The Calypso/ASX complex was then further demonstrated to function as a Polycomb Repressive-Deubiquitinase (PR-DUB) of Drosophila histone H2AK118 via its binding at the Polycomb response elements (PREs) of PcG targeted genes in Drosophila ${ }^{8}$. Consequently, mutations in Calypso that disrupt H2A deubiquitinase activity impair the repression of Hox genes in Drosophila, suggesting a critical function for the Calypso/ASX complex in transcriptional regulation and development ${ }^{8}$.

In human cells, further advancements were made by a cluster of studies that have identified subunits associated with transcriptional regulatory functions that can form complexes with BAP1, such as the transcriptional coregulator host cell factor-1 (HCF-1) $)^{9,10}$, the transcription factor Yin Yang $1(\mathrm{YY} 1)^{11}$, and the multifaceted transcription factor forkhead box proteins K1/2 (FOXK1/2) ${ }^{12}$ (Fig. 1B). From a different study, these findings were expanded upon through methods involving purification of endogenous BAP1, which showed that BAP1 had stable protein-protein interactions with HCF-1, along with four
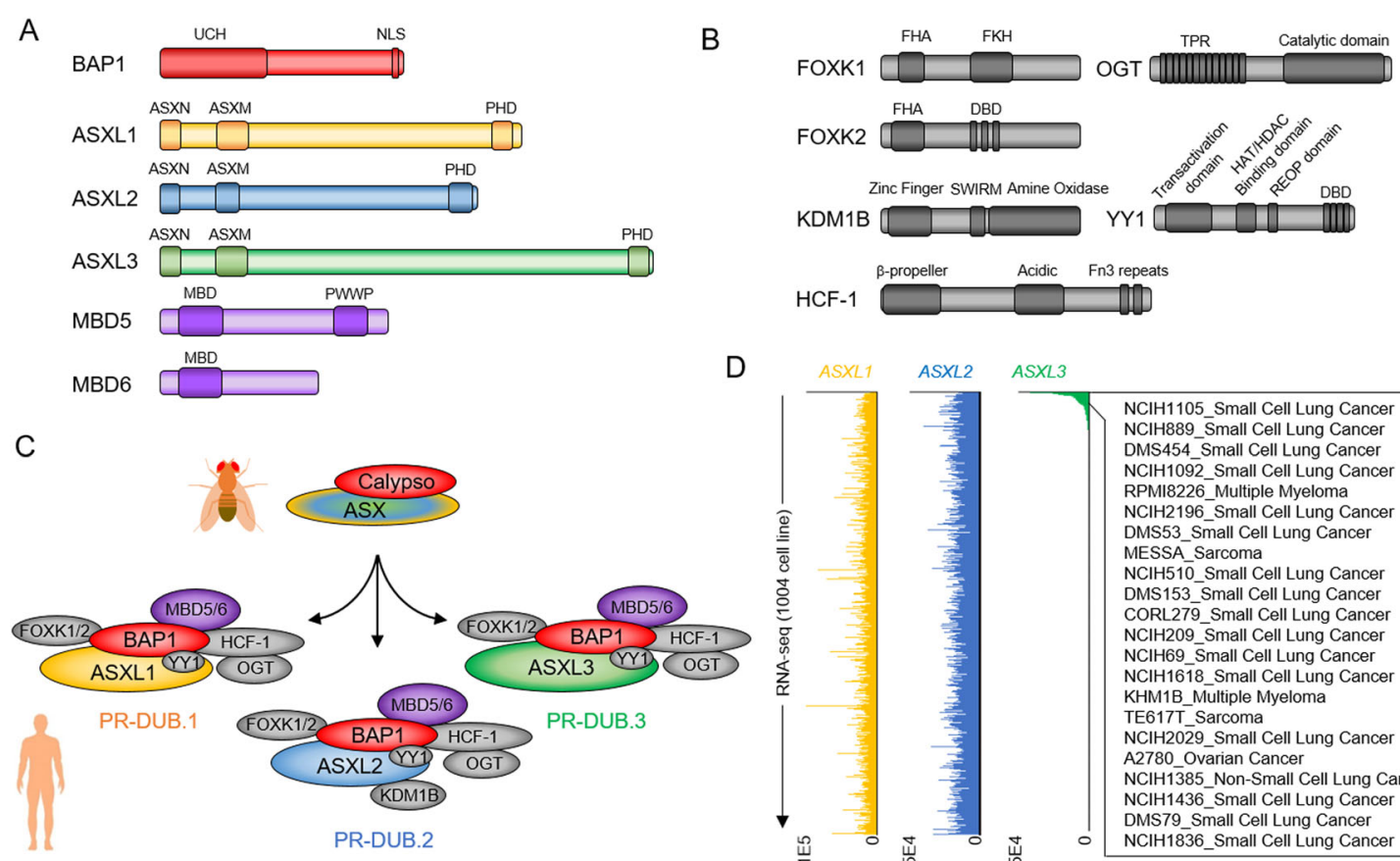

D

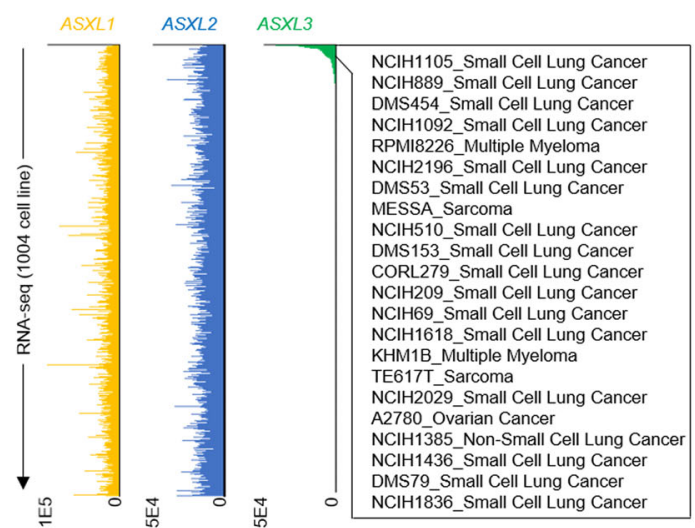

Fig. 1 Compositions of the Polycomb repressive deubiquitinase PR-DUB (BAP1) complex. A Domain organization of human BAP1, ASXL1, ASXL2, ASXL3, MBD5, and MBD6 proteins. B Domain organization of human FOXK1, FOXK2, KDM1B, HCF-1, OGT, and YY1 proteins. C Compositions of the Drosophila Calypso/ASX complex and human PR-DUB.1, PR-DUB.2, and PR-DUB.3 BAP1 complexes. D The counts per million (CPM) value for ASXL1/2/3 expression in 1,004 human cell lines. 


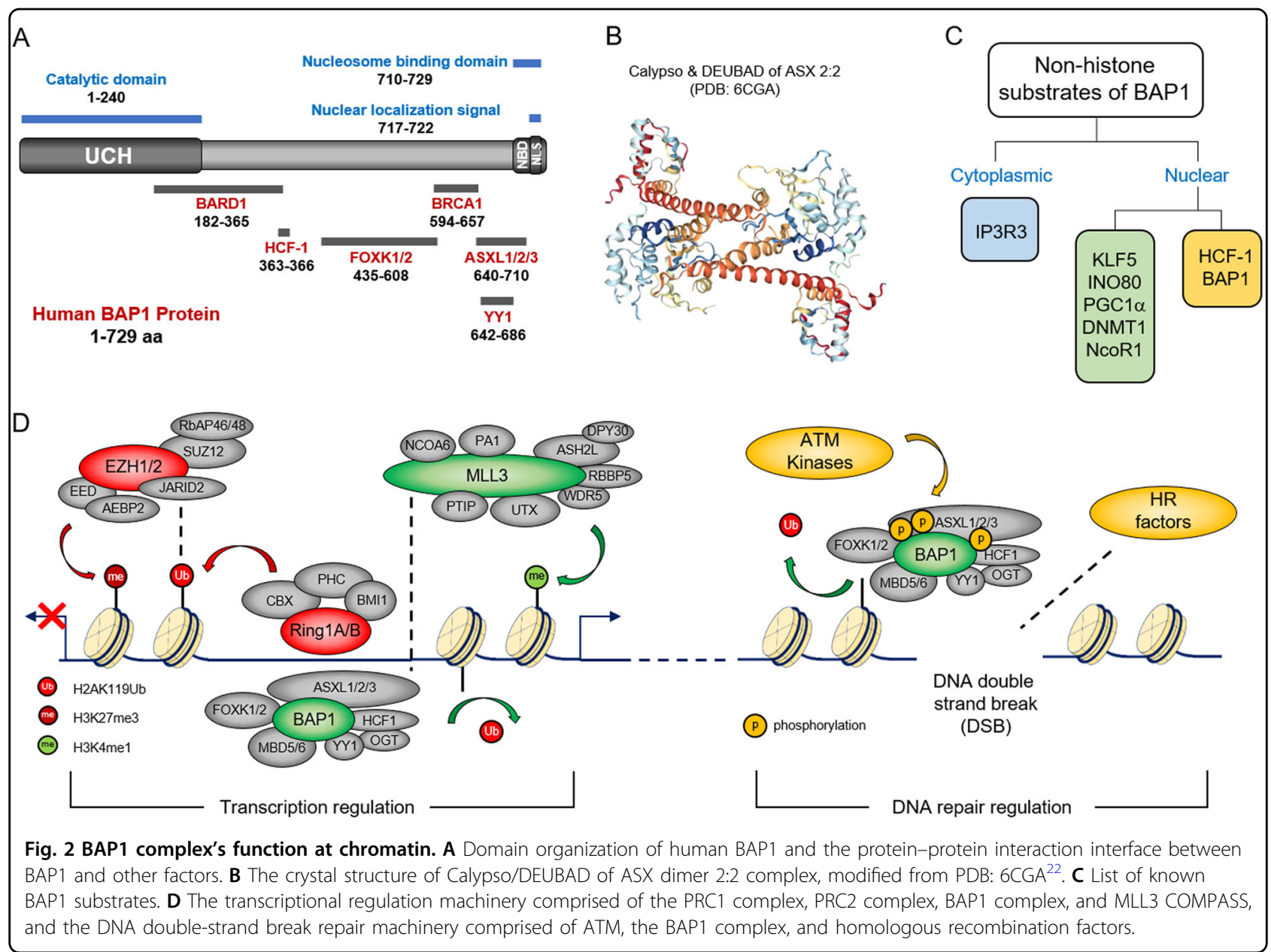

epigenetic-related subunits: $\mathrm{O}$-linked- $\mathrm{N}$-acetylglucosamine transferase (OGT), lysine-specific histone demethylase 1B (KDM1B or LSD2), and putative PcG proteins called additional sex combs-like 1/2 (ASXL1/2) ${ }^{13}$. In subsequent studies, two members of the methyl-CpG-binding domain family $(\mathrm{MBD} 5 / 6)^{14}$ and $\mathrm{ASXL}^{15,16}$ were also shown to interact with BAP1 (Fig. 1B). However, MBD5/6 can only be pulled down by a subset of components within the BAP1 complex (BAP1, ASXL2, and KDM1B) in vitro via its MBD domain. Thus, the interactions between mammalian BAP1 with MBD5/6 would need further validation with future studies.

Based on these previous findings, the human BAP1 complex is a multiprotein complex that contains as many as ten different subunits. Although several subunits-such as FOXK1/2, KDM1B, YY1, HCF-1, and OGT-have also been characterized as components within other protein complexes (Fig. 1A, B). Interestingly, only a few subunits (e.g., ASXL1-3) are exclusive within these BAP1 complexes $^{14}$. Collectively, there should be several versions of these BAP1 complexes existing within mammalian cells ${ }^{17,18}$. The three ASXL subunits within each designated BAP1 complexes are shown to be conserved from Drosophila to human (Fig. 1C). Interestingly, different from ASXL1 and ASXL2, which are both expressed at a bulk level across most of the cell lines, the expression of ASXL3 is more tissuespecific and shown to be strongly enriched in neuroendocrine cell lines such as human small cell lung cancer cells (Fig. 1D) ${ }^{16}$. This result suggests that there is a distinctive function in the chromatin localization among BAP1-ASXL1/ASXL2/ASXL3 complexes.

\section{The functions of the BAP1 complex at the chromatin level}

As a member of the UCH family, BAP1 protein is comprised of a conserved $\mathrm{N}$-terminal catalytic domain (UCH) (1-240 aa), as well as a homology C-terminal UCHL5/UCH37-like domain (ULD) (640-710 aa) (Fig. 2A). The C-terminus of BAP1 is most likely important for the assembly and stability of the BAP1 complex, due to its protein-protein interaction interfaces with $B R C A 1^{6}$, ASXL1- $3^{19}$, and $\mathrm{YY}^{11}$ (Fig. 2A). Furthermore, there are overlapping domains at the C-terminal extension of 
BAP1, which consists of a putative nuclear localization signal (NLS) sequence and a positively charged nucleosome binding domain (NBD), that auto-recruits BAP1 to substrate nucleosomes ${ }^{19}$ (Fig. 2A). Besides these two relatively conserved, functional domains within BAP1 Nterminus and $\mathrm{C}$-terminus regions, BAP1 contains an additional $\sim 395$ amino acids. This middle proteomic region is considered to be a unique linker between $\mathrm{UCH}$ and $\mathrm{C}$-terminus domains by providing another interface between BAP1 and other factors, including $\mathrm{BARD} 1^{20}$, FOXK $1 / 2^{12}$, and HCF- $1^{10}$ (Fig. 2A). Previously, it was shown that the E2/E3 hybrid ubiquitin ligase UBE2O directly modifies BAP1 NLS, and thus depletion of UBE2O impairs proper nuclear localization of $B A P 1^{21}$. However, details pertaining to the crosstalk and molecular mechanisms between overlapping domains NLS and NBD remains unknown.

To fully understand how the BAP1 complex assembles and functions, two independent groups contributed towards the development of Drosophila Calypso/ASX crystal structures, providing details that could relate to the collaboration between mammalian ASXL proteins and BAP1 (Fig. 2B). Based on the structure published by Mace's group, they generated a model of Calypso and its activating partner ASX forming a bidentate 2:2 complex, where two Calypso/ASX heterodimers dimerize via the Calypso coiled-coil regions ${ }^{22}$. However, in the crystal structure published by Müller's group, they did not observe the 2:2 complex under their experimental conditions. Although, they did observe a dimer of heterodimers at the same interface of coiled-coil regions, but only within the asymmetric unit of their crystal structures. Therefore, the structure of a Calypso/ASX nucleosome complex has been suggested in order to clarify whether or not dimerization exists ${ }^{23}$. However, within human cells, two different groups have identified a similar complex via mass spectrometry analysis consisting of a 2:1 stoichiometry for the BAP1/ASXL1 complex ${ }^{14,19}$. Intriguingly, this may indicate for a more complex structure and/or potential regulatory mechanism involving the human BAP1 complex in vivo.

BAP1 is identified as a major deubiquitinase of H2AK119Ub among all of the 100 DUBs via a targeted, small-scale shRNA screening ${ }^{24}$. Recent CRISPR screening studies by Dixit's group further identified that depletion of PRC1 subunit Ring1B (but not Ring1A) could rescue the cell death induced by loss of BAP1 ${ }^{25}$, indicating that there is a robust and direct epigenetic dynamic occurring between the PRC1 and BAP1 complexes that require a balanced state to properly regulate gene expression and determine cell fate. Additional studies have also demonstrated that H2AK119Ub could be targeted by the binding site of the UIM domain within subunit JARID2, which can provide a stronger affinity in order to recruit the PRC2 complex and inactivate transcription (Fig. 2C) ${ }^{26-28}$. In mouse models, depletion of BAP1 results in an increase of H3K27me3 levels, and repression of PRC2 targets ${ }^{29}$. Consistent with these previous findings, depletion of BAP1 by CRISPR in human cell lines also leads to a significant increase of H3K37me3 levels ${ }^{30}$, primarily due to the loss of MLL3 COMPASS (complex of proteins associated with Set1) recruitment to BAP1-dependent enhancers. Overall, these results established an epigenetic/transcriptional balance model between PRC1/PRC2, BAP1, and COMPASS complexes, representing a mechanism of "switching on/off" targeted enhancer activity and gene expression ${ }^{31}$ (Fig. 2C). Interestingly, during the cellular response to DNA double-strand breaks (DSBs) induced by UV irradiation or DNA damaging agents $^{32}$, BAP1 could be phosphorylated by DDR kinase ATM, and then recruited to the chromatin near DSB sites $^{32,33}$. It was further demonstrated that both of the catalytic activity and the phosphorylation of BAP1 by ATM is required for efficient assembly of the homologous recombination (HR) factors BRCA1 and RAD51 at the DSB loci (Fig. 2C) 34 $^{34}$ Thus, how BAP1 was recruited to the DSB site, and determining which subunit within these BAP1 complexes can mediate this function may need to be further investigated.

Besides the removal of mono-ubiquitin from histone H2AK119Ub, BAP1 can also cleave more massive Ub derivatives due to its relatively longer active-site crossover loop (usually $>14$ residues) compared to other $\mathrm{UCHs}-$ such as single-domain enzymes UCHL1/3 ${ }^{35}$ (Fig. 2B). Therefore, the length of the active-site crossover loop of UCHs determines catalytic activity and substrate specificity of ubiquitin chains and thus provides BAP1 with the potential to hydrolyze a wide range of $\mathrm{Ub}$ derivatives, including isopeptide $\mathrm{Ub}$ chains of $\mathrm{K} 48-\mathrm{diUb}^{36}$ and other non-histone substrates. For instance, one of the subunits within the BAP1 complex, HCF-1, demonstrated being the first non-histone substrate of BAP1 (Fig. 2D). BAP1 was shown to deubiquitinate $\mathrm{HCF}-1$ at the $\mathrm{N}$-terminus, which is essential for BAP1 binding and BAP1-dependent cell growth inhibition ${ }^{10}$. Other epigenetic/transcription factors such as Krueppel-like factor 5 (KLF5) ${ }^{37}$, chromatin-remodeling ATPase INO80 ${ }^{38}$, DNA methyltransferase 1 (DNMT1) ${ }^{39}$, nuclear receptor corepressor-1 $(\mathrm{NCoR} 1)^{40}$, peroxisome proliferator-activated receptor gamma coactivator 1 -alpha $(\mathrm{PGC1}-\alpha)^{41}$, and type 3 inositol-1,4,5-trisphosphate receptor (IP3R3) ${ }^{42}$ are all identified as BAP1 unique non-histone substrates (Fig. 2D). Although a conserved deubiquitination site has yet to be identified among these non-histone substrates, most of them are shown to be stabilized via deubiquitination catalyzed by BAP1. 


\section{BAP1 complex's function in metabolism}

The epigenetic balance between PRC1/PRC2, BAP1, and COMPASS complexes determines whether gene expression will be "switched on/off", indicating that BAP1 may play an important role in regulating multiple functions within different tissue types, as well as diverse cellular processes through epigenetic mechanisms (Fig. 2C). BAP1 is a ubiquitously expressed deubiquitinase; however, its potential roles are just beginning to be established in different biological processes. For instance, studies have currently linked the BAP1 complex to the maintenance of metabolic homeostasis. In addition, emerging evidence from various research groups indicated that BAP1 might have significant metabolic roles impacting other biological components while being localized within both the cytosol and nucleus (Fig. 3).

In 2012, Yang's group has identified that HCF-1, a known component of the BAP1 complex, recruits subunit OGT to O-GlcNAcylate PGC1 $\alpha$, and therefore OGlcNAcylation would facilitate the binding of deubiquitinase BAP1 in order to protect PGC1 $\alpha$ from degradation and be able to promote the metabolic pathway of gluconeogenesis. Dysregulation of gluconeogenesis is a major cause of hyperglycemia among diabetic patients, and the OGT/HCF-1/BAP1 protein complex may be a potential therapeutic target in order to improve glucose homeostasis within diabetic mice ${ }^{41}$ (Fig. 3).

More recently, the nuclear BAP1 protein complex was shown to also play an essential role in negatively regulating the expression of cystine transporter solute carrier family 7 member 11 (SLC7A11), which makes up the cystine/glutamate $\mathrm{xCT}$ amino acid antiporter and antagonizes glutamate metabolism in cells. Typically, BAP1 activates gene expression however in this particular case BAP1 represses SLC7A11 gene expression in a deubiquitinating-dependent manner by removing $\mathrm{H} 2 \mathrm{AUb}$ from the SLC7A11 promoter. Furthermore, since BAP1 inhibits cystine uptake by repressing SLC7A11 expression, it prevents glutathione (GSH) biosynthesis, leading to an increase in lipid reactive oxygen species (ROS), which

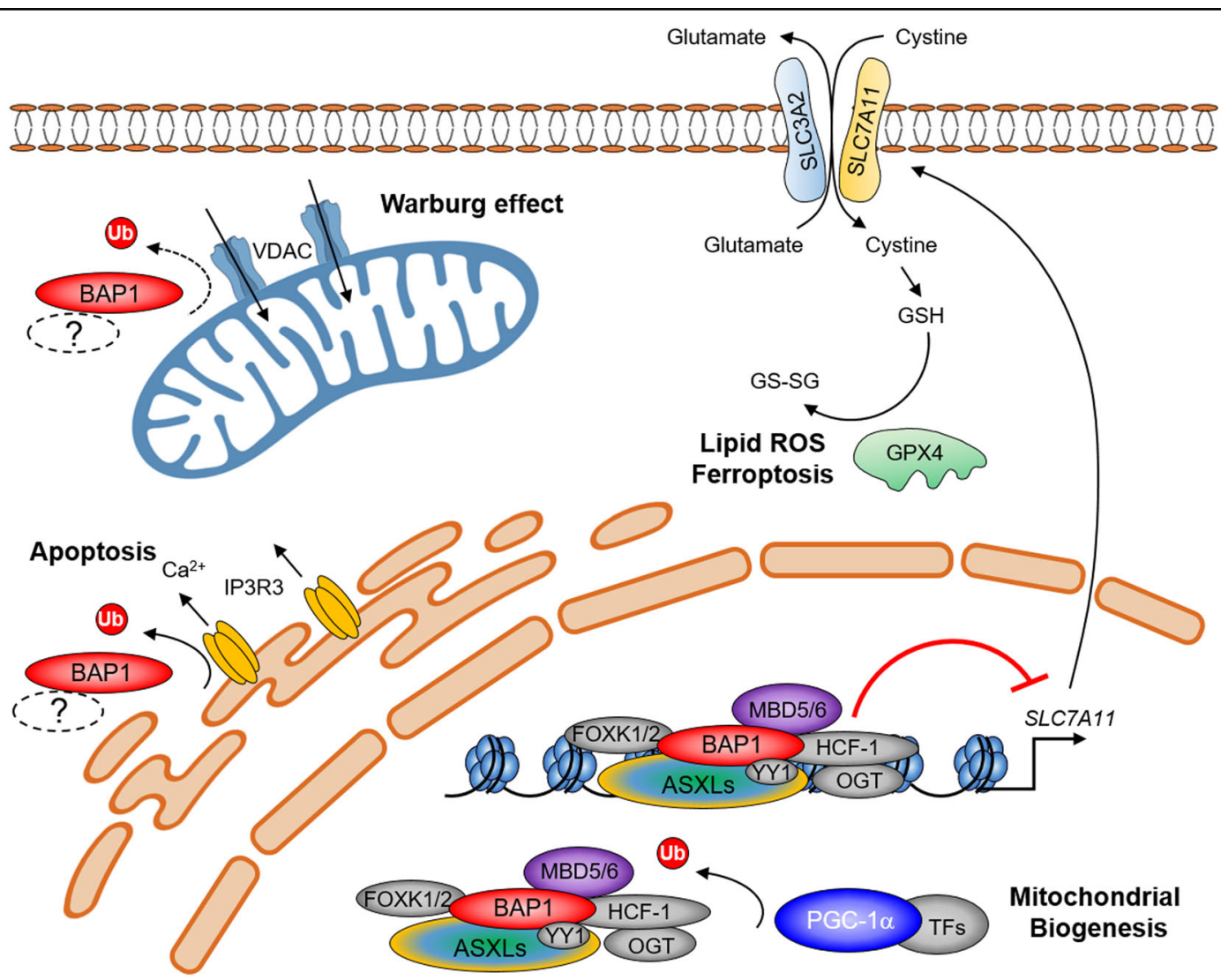

Fig. 3 BAP1 complex's function in metabolism. In nuclei, BAP1 occupies the promoter region of SLC7A11 gene, and negatively regulates the expression of SLC7A11, which mediates the uptake of extracellular cystine. Loss of BAP1 activates the glutathione biosynthesis, which is utilized by GPX4 to detoxify lipid hydroperoxide and to protect tumor cells from ferroptosis. BAP1 could also function as a deubiquitinase for non-histone substrate PGC-1a, which protects PGC-1 a from degradation and promotes gluconeogenesis. In the cytosol, BAP1 is critical for the stability of IP3R3 via deubiquitination, which mediates $\mathrm{Ca}^{2+}$ flux to mitochondria by suppressing cell transformation (as well as gatekeeper VDAC) and allowing passage of metabolites, nucleotides, and ions in/out of the mitochondria. 
promotes ferroptosis and tumor suppression ${ }^{43}$ (Fig. 3). However, the underlying mechanism of BAP1's catalytic activity-mediated transcriptional repression remains largely unexplored. It would be interesting to test whether if the transcriptional repression effect and/or H2AUb dynamics induced by loss of BAP1 could be rescued by PRC1 inhibition.

In addition, Dey's group took the advantage of developing a model involving neutron-encoded (NeuCode) lysine isotope labeling of mice, as a strategy for multiplexed proteomic analysis in BAP1-depleted mice. In their study, they observed that BAP1 deletion in the liver can potentially cause mitochondrial stress due to an increase in ubiquitination of outer mitochondrial membrane proteins, such as VDAC1/2/3 and Maob, suggesting a potential function of BAP1 in the cytosol. Moreover, the pancreas showed reduced expression of mitochondrial proteins $^{44}$. In consistent with previous results, it has been shown that normal primary cells carrying heterozygous germline BAP1 mutations in humans have been shown to increase aerobic glycolysis (also known as the "Warburg effect") and impair mitochondrial respiration/ATP production. Therefore, these results suggest that cells with BAP1 loss-of-function mutations may rely heavily on the glycolytic metabolic pathway for energy production, eventually leading to the development of one and often several malignancies ${ }^{45}$ (Fig. 3).

Initially, it was Carbone's group that provided evidence of BAP1 cytosol localization in patient-derived fibroblast cells. The results have shown that BAP1 was clearly detected as being localized at the endoplasmic reticulum (ER) by electron microscopy and immunofluorescence. In this case, BAP1 was identified as being able to bind, deubiquitylate, and stabilize type 3 inositol 1, 4, 5trisphosphate receptor (IP3R3), which modulates calcium $\left(\mathrm{Ca}^{2+}\right)$ release from the ER into the cytosol and mitochondria, promoting apoptosis. Reduced levels of BAP1 in heterozygous carriers results in a reduction of both IP3R3 and $\mathrm{Ca}^{2+}$ flux, preventing accumulated DNA damage cells from undergoing apoptosis ${ }^{42}$ (Fig. 3). Interestingly, based on the findings from a recent work published by the Margueron group, there is no detectable BAP1 protein in ASXL1/ASXL2 double knockout cells, whereas BAP1's transcript levels were unaffected ${ }^{46}$. This result suggests that BAP1 may only function within a complex with either ASXL1 and/or ASXL2 in the cytosol. However, purification of BAP1 from both nuclei and cytoplasm will be required to fully address this question.

Based on previous studies by El Bachir Affar's group, the ubiquitin-conjugating enzyme UBE2O could promote cytoplasmic localization of $\mathrm{BAP}^{21}$. It will be interesting to purify BAP1 from the cytoplasm in the absence of UBE2O. Overall, these studies have shown strong supportive data that links BAP1's functional role in several metabolic pathways, and how dysfunction can promote other pathological issues, including the development of cancer.

\section{BAP1 complex's function in the development}

Originally, PR-DUB BAP1 (Calypso) was co-purified in existence with its activating Polycomb group (PcG) protein, Additional sex combs (ASX) ${ }^{8}$, which is required for both repressive and active transcriptional states of homeotic loci ${ }^{47}$. Interestingly, an ASX mutant allele was identified to cause both anterior (regulated via PcG repressive genes) and posterior (regulated via Trithorax group (TrxG) activating genes) homeotic transformations in Drosophila. Thus, it is likely that BAP1 has an important function in embryonic development since it relies heavily on its association with ASX to protect transcriptionally active developmental genes against silencing from PRC1 ubiquitination. In Xenopus laevis development, BAP1 loss leads to transcriptional silencing of key genes regulating pluripotency-to-commitment transition into ectoderm, mesoderm, and neural crest lineages, due to lack of H3K27Ac levels accumulating at those genes loci. In mammals, the first BAP1 knockout (KO) phenotype was created in mice embryos from Dixit's group $^{13}$. They observed that BAP1 gene deletion leads to embryonic lethality due to developmental retardation between E8.5 and E9.5 days, indicating a critical role for BAP1 during embryogenesis (Table 1).

As core subunits within BAP1 complexes, all three of the ASXL proteins (ASXL1-3) were found to be essential in different developmental processes. In ASXL1 conditional-KO mice ES cells, constitutive germline loss of $A s x l 1$ also resulted in embryonic lethality (similar to $B A P 1$ depletion) as well as craniofacial abnormalities. Although some of the embryos were detected at E18.5 days, all of the pups died before birth ${ }^{48}$ (Table 1). Depletion of ASXL1 in mice could also lead to developmental abnormalities, including anophthalmia, microcephaly, cleft palates, and mandibular malformations, which are all common features associated with Bohring-Opitz syndrome among human patients. Furthermore, ASXL1 loss-of-function mutations were also observed by whole-exome sequencing in patients with Bohring-Opitz syndrome ${ }^{49}$. Recently, a de novo truncating mutation in ASXL1 was detected in an individual with abnormalities, including severe hypotonia, developmental delay, a mid-line capillary malformation, and distinctive craniofacial features ${ }^{50}$.

Comparing with ASXL1-KO mice, 50\% of ASXL2-KO mice will die before birth, and around a quarter of the pups die within two months after birth (Table 1). In addition, ASXL2-KO mice survivors had unique phenotypes distinctive from ASXL1-KO mice, including axial skeletal abnormalities, enlarged hearts, and some of them 
Table 1 BAP1 complex in development.

\begin{tabular}{|c|c|c|c|}
\hline Subunit & Phenotype in KO mice & Linked to human developmental diseases & References \\
\hline BAP1 & Developmental retardation and embryonic lethality (E8.5-E9.5 days) & - & 13 \\
\hline ASXL1 & $\begin{array}{l}\text { Embryonic lethality (>E18.5 days), dwarfism, anophthalmia, } \\
\text { microcephaly, kidney podocyte defects, and craniofacial defects }\end{array}$ & $\begin{array}{l}\text { Mutations were discovered in patients with } \\
\text { Bohring-Opitz syndrome that have a segmental } \\
\text { overgrowth }\end{array}$ & $48,75-77$ \\
\hline ASXL2 & $\begin{array}{l}>50 \% \text { pups die before birth, } 21.7 \% \text { pups die within two months, } \\
\text { axial skeletal abnormalities, enlarged heart, and reduced bone } \\
\text { mineral density (BMD) }\end{array}$ & - & 78,79 \\
\hline ASXL3 & - & $\begin{array}{l}\text { Mutations were discovered in patients with } \\
\text { Bainbridge-Ropers syndrome }\end{array}$ & 15,80 \\
\hline MBD5 & $\begin{array}{l}\text { Abnormal social behavior, cognitive impairment, and motor/ } \\
\text { craniofacial abnormalities. }\end{array}$ & $\begin{array}{l}\text { Mutations were discovered in patients with intellectual } \\
\text { disability, epilepsy, and developmental delay }\end{array}$ & $54,81-83$ \\
\hline MBD6 & - & $\begin{array}{l}\text { Mutations were discovered in patients with an autism } \\
\text { spectrum disorder }\end{array}$ & 84 \\
\hline
\end{tabular}

even have reduced bone mineral density ${ }^{51}$. These animal experiments have established separate functional roles between ASXL1 and ASXL2, in which neither of them could compensate for the function of one over the other. To date, there are no documented reports on the generation or phenotypes of ASXL3-KO mice. However, ASXL3 mutations were observed in some human neurological diseases, such as Bainbridge-Ropers syndrome and autism spectrum disorder $(\mathrm{ASD})^{15,52}$. In a recent publication using the Xenopus laevis genetic model, it was demonstrated that loss of ASXL3 protein during early embryo development highly perturbs neural cell fate specification, potentially resembling the Bainbridge-Ropers syndrome phenotype in humans ${ }^{53}$. These results from patients suggested a critical function of ASXL3 in neuronal development, differentiation, and function. Indeed, in our recent studies, we demonstrated that in comparison to ASXL1/2, ASXL3 is a more tissuespecific additional sex combs-like protein that is essential for BRD4-dependent enhancer activation in neuroendocrine cancer ${ }^{16}$.

Furthermore, BAP1 complex-specific associated proteins, MBD5/6, were also found to be involved in neurologically-related diseases. For instance, MBD5/6 mutations were found in patients with intellectual disability, epilepsy, and developmental delay ${ }^{54}$. Consistent with phenotypes observed in human patients, MBD5deficient mice also exhibited abnormal social behaviors, cognitive impairment, and motor/craniofacial abnormalities $^{55}$. Currently, MBD5/6 are known heterochromatinbinding factors. However, determining whether if MBD5/ 6 contributes to the BAP1 complex's function explicitly at the chromatin, or if it partakes in a recruitment mechanism to heterochromatin remains mostly obscure.

\section{BAP1 complex in cancer: friend or foe?}

Recent studies have shown that the dysregulation of epigenetic enzymes plays a crucial role in tumorigenesis $^{56-59}$. Both loss-of-function and gain-of-function mutations within epigenetic factors were observed in many types of human cancers ${ }^{60,61}$. BAP1 complex, which contains as many as ten different subunits (Fig. 1), is one of the most highly mutated epigenetic complexes among human cancers ${ }^{62-65}$. Different types of mutations, especially missense and truncating mutations, were observed in most of the subunits within the BAP1 complex (Fig. 4A) across different cancer types (Fig. 4B).

In BAP1, most of the somatic mutations were detected in the N-terminal UCH domain, and are identified as catalytic inactive mutations ${ }^{66}$. Interestingly, in comparison to somatic mutations, most of the germline mutation hotspots within BAP1 were located near the $\mathrm{CTD}^{67}$ (Fig. 4C). As a result, germline mutations may affect the NLS within BAP1's CTD ${ }^{21}$, which would lead to an accumulation of BAP1 in the cytosol and may then further disrupt proper metabolic processes (Fig. 3B). Moreover, germline mutations in BAP1 have also been associated with BAP1tumor predisposition syndrome that consists of multiple tumors, such as the following in descending order of frequency: uveal melanoma (UM), malignant mesothelioma (MMe), cutaneous melanoma (CM), clear cell renal cell carcinoma (ccRCC), and basal cell carcinoma $(\mathrm{BCC})^{68,69}$.

To determine the potential tumor-suppressive function of BAP1 in mammals, Dixit's group has generated the first BAP1 conditional knockout (cKO) mice. Based on their findings, BAP1-cKO mice leads to the development of splenomegaly within 4 weeks of BAP1 depletion, and myeloid cells were significantly increased 


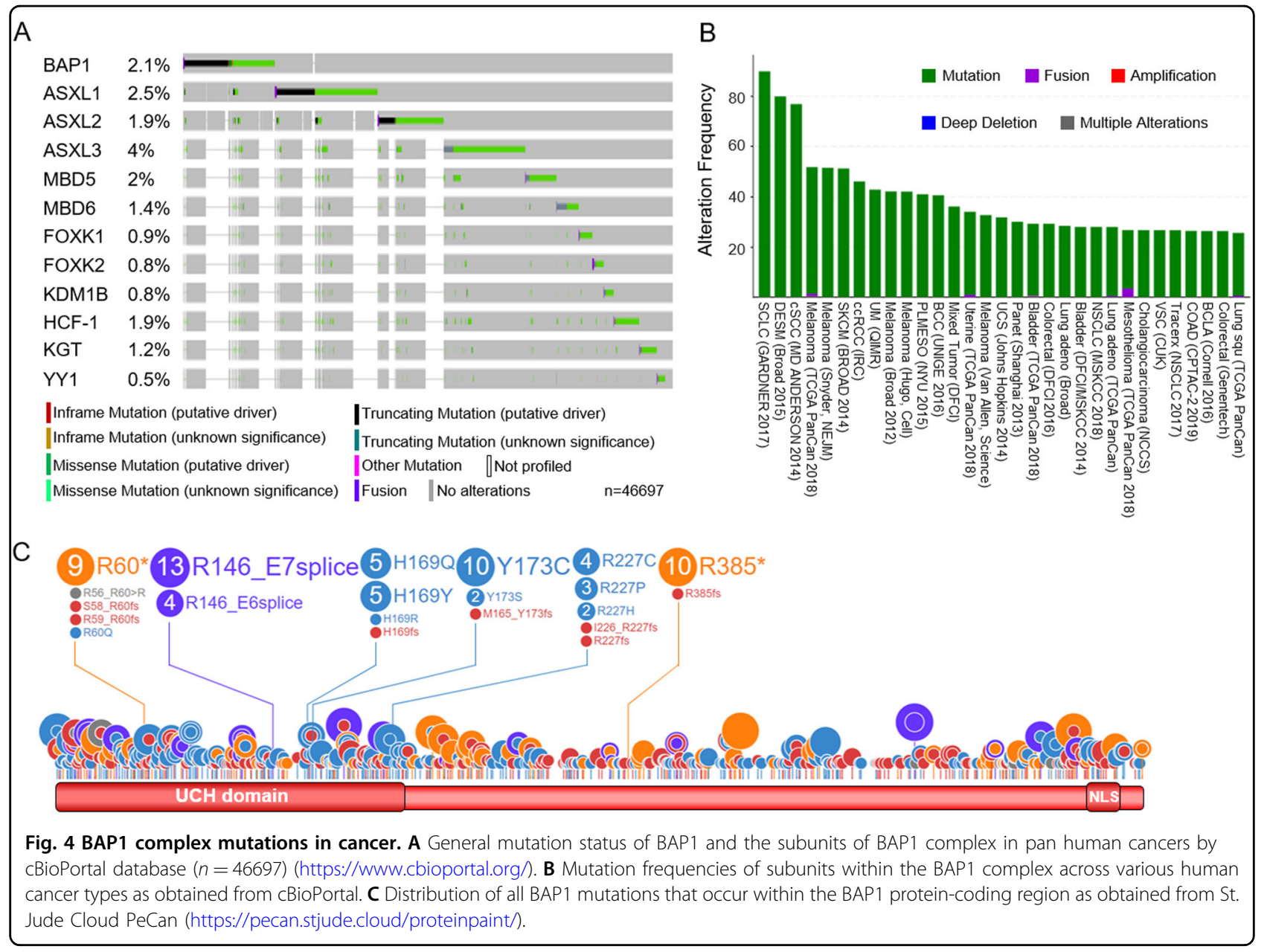

in lymph nodes and bone marrow in BAP1-cKO mice, resulting in myeloid transformation ${ }^{13}$. Mechanistically, Levine's group has further demonstrated that BAP1 loss in mice resulted in increased levels of H3K27me3 due to an elevated expression of EZH2, which leads to enhanced repression of Polycomb targeted genes ${ }^{29}$. Thus, BAP1-null tumor cells are more sensitive to EZH2 inhibitor treatment in vivo. In addition, our previous genome-wide studies have shown that enhancers binding to the BAP1 complex are responsible for the recruitment of MLL3 COMPASS, which functions as a general tumor suppressor (Fig. 2C). Depletion of BAP1 leads to a dramatic decrease of MLL3 COMPASS and its subunit, H3K27 demethylase UTX, leading to further repression of multiple tumor suppressors, such as GRHL2, RBMS3, DACT2, and DSC $3^{30}$. In melanoma, the constitutively active, oncogenic form of BRAF $\left(\mathrm{BRAF}^{\mathrm{V} 600 \mathrm{E}}\right.$ ) combined with BAP1 loss was seen in $67 \%$ of BAP1 tumor syndrome-associated lesions ${ }^{70}$. Consequently, BAP1 loss cooperates with $\mathrm{BRAF}^{\mathrm{V} 600 \mathrm{E}}$ to increase susceptibility to DNA damage, promote tumor growth, and metastasis both in vitro and in vivo ${ }^{71}$.
Although genetic depletion of BAP1 could lead to leukemogenesis in vivo, emerging studies have identified that BAP1 may also be required for tumor cell viability. In human breast cancer, BAP1 functions as deubiquitinase to stabilize the transcription factor KLF5-which is highly expressed in basal-like breast cancer-and promotes breast cancer cell proliferation, migration, and tumor growth. Furthermore, depletion of BAP1 in breast cancer cell line HCC1806 phenocopies KLF5 depletion and significantly reduced tumor growth in vivo ${ }^{37}$. In head and neck squamous cell cancer (HNSCC), BAP1-mediated histone H2AK119 mono-ubiquitination was found to be involved in radioresistance. The expression levels of BAP1 was significantly associated with poor prognosis in patients with HNSCC. This study reveals that BAP1 may be a potential therapeutic target in HNSCC clinical treatment ${ }^{72}$. In fact, based on a recent study by Yang's group, a proper level of BAP1 expression/activity is critical for the maintenance of cell fate. In terms of leukemogenesis, hyperactivation of BAP1 was observed in ASXL1 truncated leukemia cells. In addition, BAP1 hemizygous deletion in ASXL1 ${ }^{\mathrm{Y} 588 \mathrm{X}} \mathrm{Tg}$ mice-which 
have impaired hematopoietic stem/progenitor cell function and diverse myeloid malignancies-is sufficient to prevent mutant ASXL1-driven based myeloid differentiation and myeloid malignancy ${ }^{73}$. Collectively, these studies have shown the dichotomic functional role of BAP1 in different cancer types, as a tumor suppressor and/or tumor promoter, which further supports the notion of BAP1's complexity.

\section{Future directions}

To date, the BAP1 complex was discovered for over two decades now and has been actively investigated throughout the years, with emerging studies unfolding its complex nature $^{74}$. As a general transcriptional activator and a major deubiquitinase of histone H2AK119, the BAP1 complex establishes an epigenetic balance with the PRC1 complex to determine the "switching on/off" of transcriptional activity, ultimately affecting targeted gene expression. Besides histone H2AK119, a remarkable group of non-histone substrates of BAP1 has also been identified, which all play a critical role in a variety of biological processes, including metabolism and development. Dysregulation or mutations in the BAP1 complex was demonstrated to be critical during tumorigenesis. Due to the number of possible conformations and the functional complexity of this multiprotein complex, the BAP1 complex regulation and the factor that determines its chromatin binding or cytoplasmic localization remains unclear. In fact, loss-of-function and gain-of-function mutations in the BAP1 complex have both been recognized as potential drivers in different disease models, especially cancers ${ }^{65,69}$. Nevertheless, it seems that the proper activity and localization of BAP1 is essential for the determinant of cell fate. Thus, the development of smallmolecule modulators of BAP1 will be required for future therapeutic studies to further investigate its complexity and be able to potentially develop effective clinical treatments.

\section{Conflict of interest}

The authors declare that they have no conflict of interest.

\section{Publisher's note}

Springer Nature remains neutral with regard to jurisdictional claims in published maps and institutional affiliations.

Received: 3 October 2020 Revised: 28 October 2020 Accepted: 30 November 2020

Published online: 22 January 2021

\section{References}

1. Goldstein, G. et al. Isolation of a polypeptide that has Iymphocytedifferentiating properties and is probably represented universally in living cells. Proc. Natl Acad. Sci. USA 72, 11-15 (1975).
2. Weissman, A. M. Themes and variations on ubiquitylation. Nat. Rev. Mol. Cell Bio 2, 169-178 (2001).

3. Sowa, M. E., Bennett, E. J., Gygi, S. P. \& Harper, J. W. Defining the human deubiquitinating enzyme interaction landscape. Cell 138, 389-403 (2009).

4. Hermanns, T. et al. A family of unconventional deubiquitinases with modular chain specificity determinants. Nat. Commun. 9, 1-13 (2018).

5. Nijman, S. M. B. et al. A genomic and functional inventory of deubiquitinating enzymes. Cell 123, 773-786 (2005).

6. Jensen, D. E. et al. BAP1: a novel ubiquitin hydrolase which binds to the BRCA1 RING finger and enhances BRCA1-mediated cell growth suppression. Oncogene 16, 1097-1112 (1998).

7. Wilkinson, K. D. et al. The neuron-specific protein Pgp-9.5 is a ubiquitin carboxyl-terminal hydrolase. Science 246, 670-673 (1989).

8. Scheuermann, J. C. et al. Histone H2A deubiquitinase activity of the Polycomb repressive complex PR-DUB. Nature 465, 243-247 (2010).

9. Misaghi, S. et al. Association of C-terminal ubiquitin hydrolase BRCA1associated protein 1 with cell cycle regulator host cell factor 1. Mol. Cell Biol. 29, 2181-2192 (2009).

10. Machida, Y. J., Machida, Y., Vashisht, A. A., Wohlschlegel, J. A. \& Dutta, A. The deubiquitinating enzyme BAP1 regulates cell growth via interaction with HCF1. J. Biol. Chem. 284, 34179-34188 (2009).

11. $\mathrm{Yu}, \mathrm{H}$. et al. The ubiquitin carboxyl hydrolase BAP1 forms a ternary complex with YY1 and HCF-1 and is a critical regulator of gene expression. Mol. Cell Biol. 30, 5071-5085 (2010).

12. Okino, Y., Machida, Y., Frankland-Searby, S. \& Machida, Y. J. BRCA1-associated protein 1 (BAP1) deubiquitinase antagonizes the ubiquitin-mediated activation of Foxk2 target genes. J. Biol. Chem. 290, 1580-1591 (2015).

13. Dey, A. et al. Loss of the tumor suppressor BAP1 causes myeloid transformation. Science 337, 1541-1546 (2012)

14. Baymaz, H. I. et al. MBD5 and MBD6 interact with the human PR-DUB complex through their methyl-CpG-binding domain. Proteomics 14 2179-2189 (2014).

15. Srivastava, A. et al. De novo dominant ASXL3 mutations alter H2A deubiquitination and transcription in Bainbridge-Ropers syndrome. Hum. Mol. Genet. 25, 597-608 (2016)

16. Szczepanski, A. P. et al. ASXL3 bridges BRD4 to BAP1 complex and governs enhancer activity in small cell lung cancer. Genome Med. 12, 63 (2020).

17. Campagne, A. et al. BAP1 complex promotes transcription by opposing PRC1mediated H2A ubiquitylation. Nat. Commun. 10, 1-15 (2019).

18. Hauri, S. et al. A high-density map for navigating the human polycomb complexome. Cell Rep. 17, 583-595 (2016).

19. Sahtoe, D. D., van Dijk, W. J., Ekkebus, R., Ovaa, H. \& Sixma, T. K. BAP1/ASXL1 recruitment and activation for H2A deubiquitination. Nat. Commun. 7, 1-13 (2016).

20. Nishikawa, H. et al. BRCA1-associated protein 1 interferes with BRCA1/BARD1 RING heterodimer activity. Cancer Res. 69, 111-119 (2009).

21. Mashtalir, N. et al. Autodeubiquitination protects the tumor suppressor BAP1 from cytoplasmic sequestration mediated by the atypical ubiquitin ligase UBE2O. Mol. Cell 54, 392-406 (2014).

22. Foglizzo, M. et al. A bidentate Polycomb Repressive-Deubiquitinase complex is required for efficient activity on nucleosomes. Nat. Commun. 9, https://doi. org/10.1038/s41467-018-06186-1 (2018).

23. De, I. et al. Structural basis for the activation of the deubiquitinase calypso by the polycomb protein ASX. Structure 27, 528-536 e524 (2019).

24. Daou, S. et al. The BAP1/ASXL2 histone H2A deubiquitinase complex regulates cell proliferation and is disrupted in cancer. J. Biol. Chem. 290, 28643-28663 (2015).

25. He, M. et al. Intrinsic apoptosis shapes the tumor spectrum linked to inactivation of the deubiquitinase BAP1. Science 364, 283-285 (2019).

26. van Mierlo, G., Veenstra, G. J. C., Vermeulen, M. \& Marks, H. The complexity of PRC2 subcomplexes. Trends Cell Biol. 29, 660-671 (2019).

27. Tamburri, S. et al. Histone H2AK119 mono-ubiquitination is essential for polycomb-mediated transcriptional repression. Mol. Cell 77, 840-856 e845 (2020).

28. Kalb, R. et al. Histone $\mathrm{H}_{2} \mathrm{~A}$ monoubiquitination promotes histone $\mathrm{H3}$ methylation in Polycomb repression. Nat. Struct. Mol. Biol. 21, 569-571 (2014).

29. LaFave, L. M. et al. Loss of BAP1 function leads to EZH2-dependent transformation. Nat. Med. 21, 1344-1349 (2015).

30. Wang, L. et al. Resetting the epigenetic balance of Polycomb and COMPASS function at enhancers for cancer therapy. Nat. Med. 24, 758-769 (2018). 
31. Schuettengruber, B., Bourbon, H. M., Di Croce, L. \& Cavalli, G. Genome regulation by polycomb and trithorax: 70 years and counting. Cell 171, 34-57 (2017).

32. $\mathrm{Yu}, \mathrm{H}$. et al. Tumor suppressor and deubiquitinase BAP1 promotes DNA double-strand break repair. Proc. Natl Acad. Sci. USA 111, 285-290 (2014).

33. Ismail, I. H. et al. Germline mutations in BAP1 impair its function in DNA double-strand break repair. Cancer Res. 74, 4282-4294 (2014).

34. Carbone, M. et al. Tumour predisposition and cancer syndromes as models to study gene-environment interactions. Nat. Rev. Cancer 20, 533-549 (2020).

35. Larsen, C. N., Krantz, B. A. \& Wilkinson, K. D. Substrate specificity of deubiquitinating enzymes: ubiquitin C-terminal hydrolases. Biochemistry 37, 3358-3368 (1998).

36. Zhou, Z. R., Zhang, Y. H., Liu, S., Song, A. X. \& Hu, H. Y. Length of the active-site crossover loop defines the substrate specificity of ubiquitin C-terminal hydrolases for ubiquitin chains. Biochem. J. 441, 143-149 (2012).

37. Qin, J. et al. BAP1 promotes breast cancer cell proliferation and metastasis by deubiquitinating KLF5. Nat. Commun. 6, 8471 (2015).

38. Lee, H. S. et al. BAP1 promotes stalled fork restart and cell survival via INO80 in response to replication stress. Biochem. J. 476, 3053-3066 (2019).

39. Ding, $H$. et al. Systematic analysis of drug vulnerabilities conferred by tumor suppressor loss. Cell Rep. 27, 3331-3344 (2019).

40. $\mathrm{Yu}, \mathrm{L}$. et al. BAP1 regulation of the key adaptor protein NCoR1 is critical for gamma-globin gene repression. Genes Dev. 32, 1537-1549 (2018).

41. Ruan, H. B. et al. O-GlcNAc transferase/host cell factor $\mathrm{C} 1$ complex regulates gluconeogenesis by modulating PGC-1alpha stability. Cell Metab. 16, 226-237 (2012).

42. Bononi, A. et al. BAP1 regulates IP3R3-mediated $\mathrm{Ca}(2+)$ flux to mitochondria suppressing cell transformation. Nature 546, 549-553 (2017).

43. Zhang, R., He, X. H., Lin, H. Y. \& Yang, X. H. Bainbridge-Ropers syndrome with ASXL3 gene variation in a child and literature review. Zhonghua Er Ke Za Zhi 56, 138-141 (2018).

44. Baughman, J. M. et al. NeuCode proteomics reveals Bap1 regulation of metabolism. Cell Rep. 16, 583-595 (2016).

45. Bononi, A. et al. Germline BAP1 mutations induce a Warburg effect. Cell Death Differ. 24, 1694-1704 (2017).

46. Campagne, A. et al. BAP1 complex promotes transcription by opposing PRC1mediated H2A ubiquitylation. Nat. Commun. 10, 348 (2019).

47. Milne, T. A., Sinclair, D. A. R. \& Brock, H. W. The additional sex combs gene of Drosophila is required for activation and repression of homeotic loci, and interacts specifically with Polycomb and super sex combs. Mol. Gen. Genet. 261, 753-761 (1999).

48. Abdel-Wahab, O. et al. Deletion of Asxl1 results in myelodysplasia and severe developmental defects in vivo. J. Exp. Med. 210, 2641-2659 (2013).

49. Leon, E. et al. Extending the phenotypic spectrum of Bohring-Opitz syndrome: Mild case confirmed by functional studies. Am. J. Med. Genet. A 182, 201-204 (2020).

50. Efthymiou, S. et al. A de novo truncating mutation in ASXL1 associated with segmental overgrowth. J. Genet. 98, 108 (2019).

51. Baskind, H. A. et al. Functional conservation of Asxl2, a murine homolog for the Drosophila enhancer of trithorax and polycomb group gene Asx. PLoS ONE 4, e4750 (2009)

52. Kuechler, A. et al. Bainbridge-Ropers syndrome caused by loss-of-function variants in ASXL3: a recognizable condition. Eur. J. Hum. Genet. 25, 183-191 (2017).

53. Lichtig, $\mathrm{H}$. et al. Modeling Bainbridge-Ropers syndrome in Xenopus laevis embryos. Front. Physiol. 11, 75 (2020).

54. Mullegama, S. V. \& Elsea, S. H. Clinical and molecular aspects of MBD5associated neurodevelopmental disorder (MAND). Eur. J. Hum. Genet. 24, 1235-1243 (2016).

55. Camarena, V. et al. Disruption of Mbd5 in mice causes neuronal functional deficits and neurobehavioral abnormalities consistent with 2q23.1 microdeletion syndrome. EMBO Mol. Med. 6, 1003-1015 (2014).

56. Piunti, A. et al. Therapeutic targeting of polycomb and BET bromodomain proteins in diffuse intrinsic pontine gliomas. Nat. Med. 23, 493-500 (2017).

57. Piunti, A. \& Shilatifard, A. Epigenetic balance of gene expression by Polycomb and COMPASS families. Science 352, aad9780 (2016).
58. Zhao, Z. \& Shilatifard, A. Epigenetic modifications of histones in cancer. Genome Biol. 20, 245 (2019).

59. Wang, L. \& Shilatifard, A. UTX mutations in human cancer. Cancer Cell 35, 168-176 (2019).

60. Kadoch, C. \& Crabtree, G. R. Reversible disruption of mSWI/SNF (BAF) complexes by the SS18-SSX oncogenic fusion in synovial sarcoma. Cell 153, 71-85 (2013).

61. Zhu, J. et al. Gain-of-function p53 mutants co-opt chromatin pathways to drive cancer growth. Nature 525, 206-211 (2015).

62. Bejar, R. et al. Clinical effect of point mutations in myelodysplastic syndromes. N. Engl. J. Med. 364, 2496-2506 (2011).

63. Horne, G., Brewin, J. \& Chevassut, T. Genetic profiling in acute myeloid leukemia. N. Engl. J. Med. 366, 2321 (2012).

64. Carbone, M. et al. Biological mechanisms and clinical significance of BAP1 mutations in human cancer. Cancer Discov. 10, 1103-1120 (2020).

65. Okonska, A. \& Felley-Bosco, E. BAP1 missense mutations in cancer: friend or foe? Trends Cancer 5, 659-662 (2019).

66. Bhattacharya, S., Hanpude, P. \& Maiti, T. K. Cancer associated missense mutations in BAP1 catalytic domain induce amyloidogenic aggregation: a new insight in enzymatic inactivation. Sci. Rep. 5, 18462 (2015).

67. Carbone, M. et al. BAP1 and cancer. Nat. Rev. Cancer 13, 153-159 (2013).

68. Pilarski, R., Rai, K., Cebulla, C. \& Abdel-Rahman, M. BAP1 Tumor Predisposition Syndrome. In: Adam, M.P., Ardinger, H.H., Pagon, R.A., et al., eds. GeneReviews. [Internet]. Seattle (WA): University of Washington, Seattle; 1993-2021. Available from: https://www.ncbi.nlm.nih.gov/books/NBK390611/. (2016).

69. Kobrinski, D. A., Yang, H. \& Kittaneh, M. BAP1: role in carcinogenesis and clinical implications. Transl. Lung Cancer Res. 9, S60-S66 (2020).

70. Pins, A., Mihm, M. C. \& Hoang, M. P. BAP1 and BRAFV600E expression in benign and malignant melanocytic proliferations. Hum. Pathol. 46, 239-245 (2015).

71. Webster, J. D. et al. The tumor suppressor BAP1 cooperates with BRAFV600E to promote tumor formation in cutaneous melanoma. Pigm. Cell Melanoma $R$. 32, 269-279 (2019).

72. Liu, X. Y. et al. BAP1 is a novel target in HPV-negative head and neck cancer Clin. Cancer Res. 24, 600-607 (2018).

73. Guo, Y. et al. Reduced BAP1 activity prevents ASXL1 truncation-driven myeloid malignancy in vivo. Leukemia 32, 1834-1837 (2018).

74. Louie, B. H. \& Kurzrock, R. BAP1: Not just a BRCA1-associated protein. Cancer Treat. Rev. 90, 102091 (2020).

75. Bedoukian, E., Copenheaver, D., Bale, S. \& Deardorff, M. Bohring-Opitz syndrome caused by an ASXL1 mutation inherited from a germline mosaic mother. Am. J. Med. Genet. Part A 176, 1249-1252 (2018).

76. Leon, E. et al. Extending the phenotypic spectrum of Bohring-Opitz syndrome: mild case confirmed by functional studies. Am. J. Med. Genet. Part A https:/doi. org/10.1002/ajmg.a.61397 (2019).

77. Efthymiou, S. et al. A de novo truncating mutation in ASXL1 associated with segmental overgrowth. J. Genet. 98, 108 (2019).

78. Baskind, H. A. et al. Functional conservation of Asxl2, a murine homolog for the drosophila enhancer of trithorax and polycomb group gene Asx. PLoS ONE 4, e4750 (2009).

79. Farber, C. R. et al. Mouse genome-wide association and systems genetics identify Asxl2 as a regulator of bone mineral density and osteoclastogenesis. PLoS Genet. 7, e1002038 (2011).

80. Hori, I. et al. Novel splicing mutation in the ASXL3 gene causing BainbridgeRopers syndrome. Am. J. Med. Genet. Part A 170, 1863-1867 (2016).

81. Han, J. Y. et al. Diagnostic exome sequencing identifies a heterozygous MBD5 frameshift mutation in a family with intellectual disability and epilepsy. Eur. J. Med. Genet. 60, 559-564 (2017).

82. Chung, B. H. Y. et al. 2 q23 de novo microdeletion involving the MBD5 gene in a patient with developmental delay, postnatal microcephaly and distinct facial features. Am. J. Med. Genet. Part A 155a, 424-429 (2011).

83. Williams, S. R. et al. Haploinsufficiency of MBD5 associated with a syndrome involving microcephaly, intellectual disabilities, severe speech impairment, and seizures. Eur. J. Hum. Genet. 18, 436-441 (2010).

84. Cukier, H. N. et al. The expanding role of MBD genes in autism: identification of a MECP2 duplication and novel alterations in MBD5, MBD6, and SETDB1. Autism Res. 5, 385-397 (2012). 